\title{
LES EFFETS DE L'INANITION ET DE L'OXYGÉNATION SUR LA SYMBIOSE ENTRE LES TERMITES ET LEURS FLAGELLÉS INTESTINAUX
}

\author{
Par L.-R. CLEVELAND
}

Tous les termites xylophages stricts hébergent une faune extrêmement abondante de protozoaires, dont la suppression entraîne pour eux la mort en 3 à 4 semaines; à moins qu'on ne les réinfecte. Ces protozoaires peuvent être tous détruits par l'oxygénation ; d'autre part, par l'inanition, beaucoup d'entre eux périssent plusieurs jours avant leur hôte. La combinaison de ces deux méthodes donne des résultats intéressants. On peut ainsi obtenir des termites entièrement stérilisés et d'autres hébergeant sept combinaisons différentes de protozoaires, comme on le voit dans le tableau suivant :

Résultats de diverses méthodes employées pour stériliser une ou plusieurs espèces de protozoaires chez le grand termite de la côte du Pacifique: Termopsis nevadensis Hagen.

Chaque termite présente naturellement 4 espèces de protozoaires.

\begin{tabular}{|c|c|c|c|c|}
\hline MÉThode de trattement & \multicolumn{4}{|c|}{$\begin{array}{l}\text { PROTOZOAIRES PAR ORDRE } \\
\text { DE GRANDEUR }\end{array}$} \\
\hline ? & 1 & 2 & 3 & 4 \\
\hline $\begin{array}{l}\text { - signifie absence de l'espèce donnée, c'est-à dire } \\
\text { efficacité du traitement. }\end{array}$ & ฐ్๊ & జ & $\Xi$ & $\stackrel{\mathscr{g}}{\tilde{\Xi}}$ \\
\hline $\begin{array}{l}\text { + signifie présence de l'espèce, c'est-à-dire } \\
\text { traitement peu efficace ou nul. }\end{array}$ & 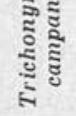 & 胥 & 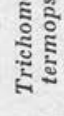 & $\begin{array}{l}\tilde{\Xi} \\
5 \\
5 \\
\vdots \\
5\end{array}$ \\
\hline 1. 6 jours de jeûne & - & + & + & + \\
\hline 2. 8 jours de jeûne ............... & - & - & + & + \\
\hline 3. $24 \mathrm{~h}$. d'oxygène à $1 \mathrm{~atm} . \ldots \ldots \ldots$. & + & t & - & + \\
\hline 4. 24 h. d'oxygène à $1 \mathrm{~atm}$. et 6 jours de jeûne & - & + & - & + \\
\hline 5. $24 \mathrm{~h}$. d'oxygène à $1 \mathrm{~atm}$. et 8 jours de jeûne & - & - & - & + \\
\hline 6. $7 \mathrm{~h}$. d'oxygène à $1 \mathrm{~atm} .1 / 2 \ldots \ldots \ldots$ & + & + & - & - \\
\hline 7. 7 h. d'oxygène à $1 \mathrm{~atm} .1 / 2$ et 6 jours de jeûne & - & + & - & - \\
\hline 8. $72 \mathrm{~h}$. d'oxygène à $1 \mathrm{~atm} . \ldots \ldots \ldots \ldots \ldots$ & - & - & - & - \\
\hline ) $9 \mathrm{~h}$. d'oxygène à $1 \mathrm{~atm} .1 / 2 \ldots \ldots \ldots$. & - & - & - & 一 \\
\hline 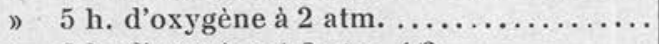 & - & - & - & - \\
\hline » $2 \mathrm{~h}$. d'oxygène à 2 atm. $1 / 2 \ldots \ldots \ldots \ldots$ & - & - & - & - \\
\hline 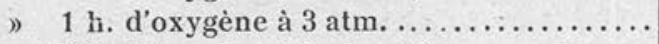 & - & - & - & - \\
\hline ) $1 / 2$ h. d'oxygène à 3 atm. $1 / 2 \ldots \ldots \ldots \ldots \ldots$ & - & - & - & - \\
\hline
\end{tabular}

Annales de Parasitologie, $\mathrm{T}$. III, $\mathrm{N}^{\circ}$ 1. - Janvier 1925, p. 35-36. 
Les traitements qui stérilisent entièrement ou partiellement les termites ne produisent pas de troubles sur eux et il a été ainsi possible d'étudier soigneusement la relation de chaque espèce de protozoaire avec son hôte et les autres parasites que ce dernier héberge. Trichonympha et Leidyopsis permettent, l'un et l'autre, à leur hôte, de vivre indéfiniment, mais Trichomonás seul ne leur permet pas une survie dépassant 60 à 70 jours en moyenne ; quant au Streblomastix, il n'a aucune influence sur la vie de son hôte; son existence comme celle du termite dépend de la présence des autres protozoaires. Quand on fait disparaître les Trichonympha, les Leidyopsis seuls se multiplient plus activement et remplacent les Trichonympha. Quand les Trichonympha et les Leidyopsis ont tous deux disparus, les Trichomonas les suppléent partiellement.

Trois autres genres de termites ont été expérimentés de la même façon. Le compte-rendu détaillé de ces expériences et d'autres est actuellement sous presse (1) et paraitra sous peu. Les protozoaires d'autres insectes, les protozoaires libres, les protozoaires parasites, en cultures, sont tous tués par l'oxygénation. Un travail est actuellement en cours concernant les effets de l'oxygène sur les protozoaires de divers animaux.

(1) Biological Bulletin.

Department of Zoology of the School of Hygiène and Public Healt-Johns Hopkins University-Baltimore U. S. A. 